\title{
Quality of life among health care workers in primary health care centres in ministry of health Jeddah 2017
}

\author{
Dalal Khaled Tashkandi ${ }^{1, *}$, Naeema Abdulkhader M Akber ${ }^{2}$ \\ ${ }^{1}$ Physician, ${ }^{2}$ Consultant, ${ }^{1}$ Dept. of Family Medicine, ${ }^{2}$ Dept. of Community Medicine, Ministry of Health, Jeddah
}

*Corresponding Author: Dalal Khaled Tashkandi

Email: dr.dalal-makki@ hotmail.com

\begin{abstract}
Objectives: To assess and identify predictors of the quality of life among health care workers in the Ministry of Health (MOH) primary health care centers in Jeddah City, 2017.

Materials and Methods: A cross-sectional study design was followed to include 489 health care workers.

Results: The mean percent score for participants' quality of life physical component summary was $64.5 \pm 19.4 \%$ that of mental component summary was $60.9 \pm 17.5 \%$, while their mean percent score of health related quality of life was $62.7 \pm 17.5 \%$. Their best attained healthstatus components were "bodily pain" and "social functioning" subscales (72.9 $\pm 23.7 \%$ and $68.4 \pm 22.3 \%$, respectively). On the other hand, their least attained health-status components were "role limitations due to emotional problems" and "energy/fatigue" $(58.7 \pm 4.7 \%$ and $56.6 \pm 18.6 \%$, respectively).

Conclusions: HRQoL of PHC providers in Jeddah City is suboptimal. The mental component of HRQoL is lower than the physical component. Some subscales of HRQoL are specially low. The main determinants of low HRQoL associated with PHC providers' personal and work characteristics include being a female, older age, having a chronic disease, serving at "Central" PHC, and being a dentistry assistant.
\end{abstract}

Keywords: Quality of Life.

\section{Introduction}

Although the quality of life assessment was almost unknown about two decades ago, it has rapidly become an integral variable of outcome in clinical research. Over 1000 new articles are indexed each year under "quality of life". Currently, the importance of quality of life is broadly acknowledged. $^{1}$

Quality of life (QOL) is one of the most important issues in every organization, especially in health organizations. When a health organization offers good quality of life to their employees, their production will improve, leading to better well-being of their healthcare workers (HCW). Eventually, they will have high commitment and ultimately reduce costs that incur due to a high level of stress. Hence, by having good quality of life, the society can enjoy increased health organizational productivity and higher opportunity for growth with better participation from $\mathrm{HCW}$ that has a significant impact on the community. Moreover, a happy $\mathrm{HCW}$ will experience positive feeling and this feeling becomes carried to their patients, family and the society. ${ }^{2}$

HCWs need to be clear about the conceptual definition of QOL and not to confound it with functional status, symptoms, disease processes, or treatment side-effects. Although the definition of QOL is still evolving, Revicki and colleagues defined QOL as "a broad range of human experiences related to one's overall well-being. It implies values, based on subjective functioning in comparison with personal expectations and is defined by subjective experiences, states, and perceptions". This definition denotes a meaning for QOL that transcends health. ${ }^{3}$

Although QOL has been the focus of attention for over a decade, only few studies were conducted in Saudi Arabia, while there are no studies done in Jeddah City. Therefore, this study is intended to give insight into the QOL among HCWs at the respective health organization. ${ }^{4}$

Thinkers have discussed the "good life" and the desirable society for millennia. In the last decades, scientists offered several alternative approaches to defining and measuring the quality of life. ${ }^{5}$ The Quality of Life Scale (QOLS), first developed by the American psychologist, John Flanagan, befits this definition of QOL.

QOL represent 5 conceptual domains of QOL that were empirically derived from the 6500 critical incidents that Flanagan and his team collected. Its scores are summed so that a higher score indicates a higher QOL. ${ }^{3}$

It also allows for objective comparisons to be made between the situations of particular groups and what is normative. Considerable agreement exists that QOL is multidimensional. ${ }^{6}$ QOL measures have become increasingly popular as outcome measures. Currently, the RAND-36 is perhaps the most widely used health-related quality of life (HRQOL) survey instrument worldwide. It is comprised of 36 items that assess eight health concepts: physical functioning, role limitations caused by physical health problems, role limitations caused by emotional problems, social functioning, emotional well-being, energy/fatigue, pain, and general health perceptions. Physical and mental health summary scores are also derived from the eight RAND-36 scales. ${ }^{7,8}$

The World Health Organization's project to develop a quality of life instrument (the WHOQOL) has been developed collaboratively in several culturally diverse centers over four years and produces a multi-dimensional profile of scores across six domains and 24 subdomains of quality of life. ${ }^{9}$ 
However, there is a critical appraisal of the quality of QOL measurements, since QOL is a uniquely personal perception, denoting the way that individual persons feel about their health status and/or nonmedical aspects of their lives. ${ }^{10-11}$

There is no consensus on what quality of life really is. Considering how multidimensional and subjective this concept is, health organizations created a different questionnaire encompassing the physical, psychological, social and environmental aspects. This questionnaire, which is today the basis for studies about individual health status, is a tool used to assess the well-being of individuals. ${ }^{12-13}$

No study has been conducted to evaluate QOL among $\mathrm{MOH} \mathrm{HCWs}$ in Jeddah. Therefore, the Investigator chose HCWs population since if they have a bad QOL, they will have a negative impact on their professional work. Moreover, the investigator has a special interest in this topic because she always fights to be able to cope with the type of work she does and to maintain a proper work-life balance. Also, she frequently comes across several experiences of HCWs having bad quality of life.

This study aimed to assess the quality of life and its predictors among HCWs in MOH PHCCs in Jeddah 2017.

\section{Materials and Methods}

A cross-sectional study design among Health care workers at MOH PHCC in Jeddah City._All PHCC workers who were present during the time of conducting the study were included: PHC physician (Dentists, GPs, family physicians \& family medicine residents), Nurses, Technicians (Lab and radiology technicians), Pharmacists, and administrative staff. While PHC physicians with less than one year experience were excluded.

According to the latest available data, the total number of HCWs in MOH PHCCs in Jeddah is 2264. The "minimum" sample size was calculated using the online Raosoft sample size calculator program (14) to be 475, based on the following data: Estimated prevalence of $50 \%$ (to maximize the sample size), Confidence level of $95 \%$, Acceptable margin of error of 4\%, Population size of 2,264.

A "multistage random sampling technique was followed. The calculated sample size was distributed over 5 sectors, according to their geographic areas, i.e., north-West, north-east, central, south-west, and south-east. Each stratum has 8-12. PHCCs.
1. $1^{\text {st }}$ stage: the investigator selected 2 PHCCs from each sector by simple random technique. This accounted for 10 PHCCs.

2. $2^{\text {nd }}$ stage: the investigator reached all HCWs in the selected PHCCs of each sector by taking all HCWs from each center. Staff were selected by proportional allocation from each PHCC.

In addition to participants' sociodemographic variables, a self-administered validated Arabic version questionnaire of the SF-36, from RAND, was used in this study. ${ }^{7}$ This standard questionnaire was used to evaluate $\mathrm{MOH}$ PHC physicians' health related quality of life (HRQoL). It consists of 36 questions that measure the HRQoL in two components, i.e., physical and mental health status, with eight subscales. The physical health domain includes physical functioning, role limitations due to physical problems, bodily pain and general health. On the other hand, the mental health component includes vitality (energy/fatigue), social functioning, role limitations due to emotional problems, and emotional wellbeing. ${ }^{15}$

Each item within the SF-36 questionnaire evaluates the positive and negative aspects of participants' health status, with a percent score that ranges from $0 \%$ (indicating worst quality) to $100 \%$ indicating the best quality of life. ${ }^{16}$

After getting all necessary official permissions from the higher authority, the investigator visited each selected $\mathrm{MOH}$ PHCC and personally distributed the questionnaire sheets to the $\mathrm{HCW}$, hand by hand during their break time and then collected the sheets at the same day, one PHCC a day.

Collected data were entered and analyzed using the Statistical Package for Social Sciences (SPSS, version 22). Descriptive statistics for the total scores and sub-scores for QOL were calculated. Categorical data were presented as frequencies and proportions, while quantitative data were presented as means and standard deviations. For comparison, t-test and one way-analysis of variance (ANOVA) for continuous data were applied. P-values < 0.05 were considered as statistically significant.

All official approvals were fulfilled, i.e., the research committee approval, higher authority, local, institutional and departmental approvals. Written or verbal consents from each participant was obtained before data collection.

\section{Results}

Table 1: Personal characteristics of study sample

\begin{tabular}{|l|c|c|}
\hline \multicolumn{1}{|c|}{ Personal characteristics } & No. & $\%$ \\
\hline Gender & & \\
\hline$\bullet \quad$ Male & 213 & 43.6 \\
\hline$\bullet \quad$ Female & 276 & 56.4 \\
\hline Age & & \\
\hline$\bullet \quad<25$ years & 23 & 4.7 \\
\hline$\bullet \quad 25-35$ years & 250 & 51.1 \\
\hline$\bullet \quad 36-45$ years & 148 & 30.3 \\
\hline$\bullet$ 46-55 years & 56 & 11.5 \\
\hline$\bullet \quad>55$ years & 12 & 2.5 \\
\hline
\end{tabular}




\begin{tabular}{|c|c|c|}
\hline \multicolumn{3}{|l|}{ Nationality } \\
\hline - $\quad$ Saudi & 451 & 92.2 \\
\hline Non-Saudi & 38 & 7.8 \\
\hline \multicolumn{3}{|l|}{ Marital status } \\
\hline - $\quad$ Single & 88 & 18.0 \\
\hline - $\quad$ Married & 347 & 71.0 \\
\hline - $\quad$ Divorced & 44 & 9.0 \\
\hline - $\quad$ Widow & 10 & 2.0 \\
\hline \multicolumn{3}{|l|}{ Number of children } \\
\hline $\begin{array}{l} \\
\end{array}$ & 148 & 30.3 \\
\hline - 1 & 72 & 14.7 \\
\hline - 2 & 84 & 17.2 \\
\hline - 3 & 96 & 19.6 \\
\hline - $4+$ & 89 & 18.2 \\
\hline \multicolumn{3}{|l|}{ Chronic diseases } \\
\hline - $\quad$ Absent & 537 & 73.2 \\
\hline - $\quad$ Present: & 131 & 26.8 \\
\hline - $\quad$ Diabetes & 53 & 10.8 \\
\hline - $\quad$ Hypertension & 58 & 11.9 \\
\hline - Dyslipidemia & 28 & 5.7 \\
\hline - Bronchial asthma & 24 & 4.9 \\
\hline - $\quad$ Others & 20 & 4.1 \\
\hline \multicolumn{3}{|l|}{ Position } \\
\hline - $\quad$ Physician & 101 & 20.7 \\
\hline - $\quad$ Dentist & 32 & 6.5 \\
\hline - $\quad$ Pharmacist & 28 & 5.7 \\
\hline - $\quad$ Technician & 83 & 17.0 \\
\hline - $\quad$ Nurse & 143 & 29.2 \\
\hline - Dentistry assistant & 14 & 2.9 \\
\hline - $\quad$ Administrator & 88 & 18.0 \\
\hline \multicolumn{3}{|l|}{ Monthly income (SR) } \\
\hline - $\quad<10,000$ & 95 & 19.4 \\
\hline - $10,000-14,999$ & 186 & 38.0 \\
\hline - $15,000-19,999$ & 123 & 25.2 \\
\hline - $20,000-24,999$ & 57 & 11.7 \\
\hline - $25,000-29,999$ & 12 & 2.5 \\
\hline - $\geq 30,000$ & 16 & 3.3 \\
\hline \multicolumn{3}{|l|}{ Experience in $\mathrm{PHC}$} \\
\hline - $\quad<5$ years & 95 & 19.4 \\
\hline - 5-9 years & 171 & 35.0 \\
\hline - $10-19$ years & 144 & 29.4 \\
\hline - $20+$ years & 79 & 16.2 \\
\hline
\end{tabular}

Table 1 shows that $56.4 \%$ of participants were females. Age of $51.1 \%$ of participants was $25-35$ years, while $30.3 \%$ of them aged 36-54 years. The majority of participants (92.2\%) were Saudi. Most participants (71\%) were married. Almost one third of participants $(30.3 \%)$ had no children, while $19.6 \%$ had 3 children and $18.2 \%$ had 4 children or more. About one fourth of participants had chronic disease, mainly diabetes $(10.8 \%)$ or hypertension $(11.9 \%)$. Less than third $29.2 \%$ of participants were nurses, $20.7 \%$ were physicians, $18 \%$ were administrators, $17 \%$ were technicians, $6.5 \%$ were dentists, $5.7 \%$ were pharmacists and $2.9 \%$ were dentistry assistants. The monthly income of $38 \%$ of participants was 10,000-14,999 SR, while $25.2 \%$ had a monthly income of 20,000-24,999 SR. more than one third of participants (35\%) had 5-9 years' experience in PHC, while $29.4 \%$ had $10-19$ years' experience. 
Table 2: Participants' percent scores (Mean \pm SD) for their health status components

\begin{tabular}{|l|c|}
\hline \multicolumn{1}{|c|}{ Health Status Components } & Mean \pm SD \\
\hline Physical component summary: & $64.5 \pm 19.4$ \\
\hline$\bullet \quad$ Physical functioning & $64.2 \pm 28.0$ \\
\hline$\bullet \quad$ Bodily Pain & $72.9 \pm 23.7$ \\
\hline$\bullet \quad$ Role limitations due to physical health & $58.7 \pm 4.7$ \\
\hline$\bullet \quad$ General Health & $62.2 \pm 15.6$ \\
\hline Mental component summary: & $60.9 \pm 17.5$ \\
\hline$\bullet \quad$ Role limitations due to emotional problems & $55.1 \pm 42.6$ \\
\hline$\bullet \quad$ Energy/fatigue & $56.6 \pm 18.6$ \\
\hline$\bullet \quad$ Social functioning & $68.4 \pm 22.3$ \\
\hline$\bullet \quad$ Emotional well-being & $63.7 \pm 18.3$ \\
\hline Health-Related Quality of Life & $62.7 \pm 17.5$ \\
\hline
\end{tabular}

Table 2 show that score for participants' quality of life physical component summary (Mean $\pm \mathrm{SD}$ ) was $64.5 \pm 19.4 \%$, that of mental component summary was $60.9 \pm 17.5 \%$, while their mean percent score of health related quality of life was $2.7 \pm 17.5 \%$. Their best attained health-status components were "bodily pain" and "social functioning" subscales $(72.9 \pm 23.7 \%$ and $68.4 \pm 22.3 \%$, respectively). On the other hand, their least attained health-status components were "role limitations due to emotional problems" and "energy/fatigue" (58.7 $\pm 4.7 \%$ and $56.6 \pm 18.6 \%$, respectively).

Table 3 shows that female participants' mean percent score for physical component summary was significantly less, i.e., worse, than that for males $(62.7 \pm 19.3$ and $66.8 \pm 19.4$, respectively, $\mathrm{p}=0.019$ ). Moreover, mean percent scores for participants' physical component summary differed significantly according to their age $(\mathrm{p}=0.022)$, with younger participants (i.e., aged $<25$ years) having the highest score. In addition, participants who had chronic diseases had significantly lower physical component summary than those who did not have chronic diseases (59.8 \pm 19.7 and 66.2 \pm 19.0 , respectively, $\mathrm{p}=0.001$ ). However, participants' physical component summary did not differ significantly according to their nationality, marital status or number of children. Participants' "physical component summary" scores differed significantly according to their geographical sector $(\mathrm{p}=0.046)$, with highest among participants at the Northwestern sector and lowest (68.1 \pm 21.2$)$ among those at Central sector (59.6 \pm 19.1$)$. Moreover, participants' "physical component summary" differed significantly according to their monthly income ( $p=0.022$ ), being best attained by those who had $\geq 30,000$ SR (70.2 \pm 20.6$)$. In addition, their scores differed significantly according to their experience in PHC $(\mathrm{p}=0.009)$, with highest scores among those with least experience (69.5 \pm 17.7$)$. However, their "physical component summary" scores did not differ significantly according to their position. Table (14) shows that female participants' mean percent score for mental component summary was significantly less, i.e., worse, than that for males $(59.2 \pm 20.2$ and 63.1 \pm 19.0 , respectively, $\mathrm{p}=0.030)$. Moreover, participants who had chronic diseases had significantly lower mental component summary than those who did not have chronic diseases $(56.4 \pm 19.8$ and $62.6 \pm 19.4$, respectively, $\mathrm{p}=0.002$ ). However, participants' mental component summary did not differ significantly according to their age, nationality, marital status or number of children. Participants' "mental component summary" scores differed significantly according to their position $(\mathrm{p}=0.028)$, being best attained by technicians $(66.0 \pm 19.0)$ and least attained by dentistry assistants (52.4 \pm 16.2$)$. However, their "mental component summary" scores did not differ significantly according to their sector, monthly income or experience in PHC. Female participants' mean percent score for "health-related quality of life" was significantly less, i.e., worse, than that for males $(61.0 \pm 17.5$

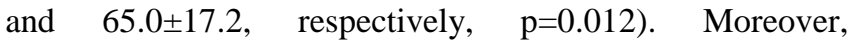
participants who had chronic diseases had significantly lower "health-related quality of life" than those who did not have chronic diseases $(58.1 \pm 18.3$ and $64.4 \pm 16.9$, respectively, $\mathrm{p}<0.001)$. However, participants' "healthrelated quality of life" did not differ significantly according to their age, nationality, marital status or number of children. Participants' "health-related quality of life" scores differed significantly according to their position $(\mathrm{p}=0.038)$, being best attained by technicians $(67.6 \pm 16.5)$ and least attained by dentistry assistants $(55.8 \pm 16.3)$. However, their "health-related quality of life" scores did not differ significantly according to their sector, monthly income or experience in PHC.

Table 3: Participants' mean percent scores of "physical component summary", "mental component summary", and "health-related quality of life" according to their personal and work characteristics

\begin{tabular}{|l|c|c|c|c|c|c|c|}
\hline $\begin{array}{c}\text { Personal and work } \\
\text { characteristics }\end{array}$ & \multirow{2}{*}{ No. } & \multicolumn{2}{c|}{$\begin{array}{c}\text { Physical component } \\
\text { summary }\end{array}$} & \multicolumn{2}{c|}{$\begin{array}{c}\text { Mental component } \\
\text { summary }\end{array}$} & \multicolumn{2}{c|}{ health-related quality of life } \\
\cline { 3 - 8 } & & Mean \pm SD & P-value & Mean \pm SD & P-value & Mean \pm SD & P-value \\
\hline Gender & & & & & & & \\
\hline Male & 213 & $66.8 \pm 19.4$ & & $63.1 \pm 19.0$ & & $65.0 \pm 17.2$ & \\
\hline
\end{tabular}




\begin{tabular}{|c|c|c|c|c|c|c|c|}
\hline - $\quad$ Female & 276 & $62.7 \pm 19.3$ & 0.019 & $59.2 \pm 20.2$ & 0.030 & $61.0 \pm 17.5$ & 0.012 \\
\hline \multicolumn{8}{|l|}{ Age } \\
\hline - $\quad<25$ years & 23 & $71.2 \pm 21.2$ & & $63.7 \pm 20.4$ & & $67.4 \pm 20.0$ & \\
\hline 25-35 years & 250 & $66.1 \pm 18.4$ & & $62.3 \pm 20.1$ & & $64.2 \pm 16.8$ & \\
\hline - $\quad 36-45$ years & 148 & $63.4 \pm 20.9$ & & $59.2 \pm 19.6$ & & $61.3 \pm 18.3$ & \\
\hline - $\quad 46-55$ years & 56 & $58.0 \pm 17.2$ & & $57.3 \pm 18.0$ & & $57.7 \pm 15.9$ & \\
\hline - $\quad>55$ years & 12 & $61.8 \pm 20.4$ & 0.022 & $64.8 \pm 22.1$ & 0.270 & $63.3 \pm 19.7$ & 0.057 \\
\hline \multicolumn{8}{|l|}{ Nationality } \\
\hline - $\quad$ Saudi & 451 & $64.8 \pm 19.2$ & & $61.3 \pm 19.8$ & & $63.1 \pm 17.3$ & \\
\hline - $\quad$ Non-Saudi & 38 & $60.3 \pm 21.5$ & 0.170 & $56.3 \pm 19.1$ & 0.134 & $58.3 \pm 18.9$ & 0.108 \\
\hline \multicolumn{8}{|l|}{ Marital status } \\
\hline - $\quad$ Single & 88 & $67.1 \pm 19.2$ & & $61.4 \pm 19.0$ & & $64.3 \pm 17.0$ & \\
\hline - $\quad$ Married & 347 & $64.6 \pm 19.2$ & & $61.3 \pm 19.6$ & & $63.0 \pm 17.2$ & \\
\hline - $\quad$ Divorced & 44 & $60.5 \pm 18.9$ & & $58.2 \pm 21.8$ & & $59.3 \pm 18.4$ & \\
\hline - $\quad$ Widow & 10 & $56.4 \pm 26.7$ & 0.162 & $54.8 \pm 25.0$ & 0.572 & $55.6 \pm 25.5$ & 0.259 \\
\hline \multicolumn{8}{|l|}{ Number of children } \\
\hline $\begin{array}{ll}-0 \\
\end{array}$ & 148 & $66.6 \pm 18.7$ & & $62.1 \pm 18.9$ & & $64.3 \pm 16.9$ & \\
\hline $\begin{array}{ll}-1 \\
\end{array}$ & 72 & $65.4 \pm 19.1$ & & $61.8 \pm 20.4$ & & $63.6 \pm 16.8$ & \\
\hline - 2 & 84 & $64.8 \pm 19.9$ & & $62.6 \pm 20.5$ & & $63.7 \pm 18.1$ & \\
\hline - 3 & 96 & $64.9 \pm 20.5$ & & $60.2 \pm 21.4$ & & $62.5 \pm 19.2$ & \\
\hline - $4+$ & 89 & $59.6 \pm 18.6$ & 0.101 & $57.5 \pm 18.2$ & 0.406 & $58.5 \pm 16.2$ & 0.147 \\
\hline \multicolumn{8}{|l|}{ Chronic diseases } \\
\hline - $\quad$ Absent & 537 & $66.2 \pm 19.0$ & & $62.6 \pm 19.4$ & & $64.4 \pm 16.9$ & \\
\hline - $\quad$ Present & 131 & $59.8 \pm 19.7$ & 0.001 & $56.4 \pm 19.8$ & 0.002 & $58.1 \pm 18.3$ & $<0.001$ \\
\hline \multicolumn{8}{|l|}{ Sector } \\
\hline - $\quad$ Northeastern & 118 & $64.7 \pm 18.7$ & & $60.1 \pm 18.9$ & & $62.4 \pm 16.7$ & \\
\hline - $\quad$ Northwestern & 96 & $68.1 \pm 21.2$ & & $63.6 \pm 23.1$ & & $65.8 \pm 20.4$ & \\
\hline - $\quad$ Southeastern & 81 & $65.5 \pm 17.3$ & & $60.2 \pm 16.8$ & & $62.8 \pm 14.9$ & \\
\hline - $\quad$ Southwestern & 98 & $64.8 \pm 19.7$ & & $60.3 \pm 20.5$ & & $62.5 \pm 17.8$ & \\
\hline - $\quad$ Central & 96 & $59.6 \pm 19.1$ & 0.046 & $60.6 \pm 19.0$ & 0.711 & $60.1 \pm 16.9$ & 0.265 \\
\hline \multicolumn{8}{|l|}{ Position } \\
\hline - $\quad$ Physician & 101 & $65.1 \pm 19.5$ & & $60.4 \pm 21.3$ & & $62.7 \pm 17.2$ & \\
\hline - $\quad$ Dentist & 32 & $64.0 \pm 18.8$ & & $61.1 \pm 19.3$ & & $62.6 \pm 17.2$ & \\
\hline - $\quad$ Pharmacist & 28 & $65.0 \pm 18.3$ & & $60.5 \pm 22.4$ & & $62.8 \pm 18.0$ & \\
\hline - $\quad$ Technician & 83 & $69.2 \pm 18.5$ & & $66.0 \pm 19.0$ & & $67.6 \pm 16.5$ & \\
\hline - $\quad$ Nurse & 143 & $62.0 \pm 20.2$ & & $57.6 \pm 20.1$ & & $59.8 \pm 18.8$ & \\
\hline - $\quad$ Dentistry assistant & 14 & $59.2 \pm 18.7$ & & $52.4 \pm 16.2$ & & $55.8 \pm 16.3$ & \\
\hline - $\quad$ Administrator & 88 & $64.4 \pm 19.1$ & 0.208 & $63.7 \pm 16.9$ & 0.028 & $64.0 \pm 16.0$ & 0.038 \\
\hline \multicolumn{8}{|l|}{ Monthly income (SR) } \\
\hline - $\quad<10,000$ & 95 & $64.7 \pm 19.9$ & & $61.2 \pm 18.5$ & & $63.3 \pm 17.1$ & \\
\hline $\begin{array}{ll} & 10,000-14,999 \\
\end{array}$ & 186 & $66.5 \pm 18.5$ & & $62.5 \pm 19.5$ & & $64.5 \pm 17.2$ & \\
\hline - $15,000-19,999$ & 123 & $64.4 \pm 19.7$ & & $58.5 \pm 21.5$ & & $61.5 \pm 18.1$ & \\
\hline - $\quad 20,000-24,999$ & 57 & $56.5 \pm 18.6$ & & $57.7 \pm 19.3$ & & $57.1 \pm 17.5$ & \\
\hline - $\quad 25,000-29,999$ & 12 & $64.1 \pm 20.1$ & & $58.1 \pm 20.4$ & & $61.1 \pm 16.9$ & \\
\hline - $\quad \geq 30,000$ & 16 & $70.2 \pm 20.6$ & 0.022 & $68.8 \pm 16.9$ & 0.189 & $69.5 \pm 15.2$ & 0.051 \\
\hline \multicolumn{8}{|l|}{ Experience in PHC } \\
\hline - $\quad<5$ years & 95 & $69.5 \pm 17.7$ & & $62.3 \pm 20.0$ & & $65.9 \pm 16.5$ & \\
\hline - 5-9 years & 171 & $64.8 \pm 19.5$ & & $61.8 \pm 20.1$ & & $63.3 \pm 17.7$ & \\
\hline - $10-19$ years & 144 & $63.5 \pm 20.5$ & & $60.5 \pm 19.5$ & & $62.0 \pm 18.0$ & \\
\hline - $20+$ years & 79 & $59.6 \pm 17.7$ & 0.009 & $58.2 \pm 19.4$ & 0.506 & $58.9 \pm 16.7$ & 0.064 \\
\hline
\end{tabular}

Table 4 shows that participants who had chronic diseases had significantly less "physical functioning" than those who did not have chronic diseases $(59.0 \pm 26.5$ and $66.1 \pm 28.3$, respectively, $\mathrm{p}=0.013$ ). However, the "physical functioning" subscale did not differ significantly according to participants' gender, age, nationality, marital status or number of children. Participants "physical functioning" differed significantly according to their monthly income, being best attained by those who had 15,000-19,999 SR, 10,000-14,999 SR and those who had $\geq 30,000$ SR $(65.0 \pm 31.1, \quad 66.9 \pm 25.4$ and $67.4 \pm 26.4$, respectively, $\mathrm{p}=0.034)$. However, the "physical functioning" subscale did not differ significantly according to geographical sector, participants' position, or their experience in PHC. Female 
participants had significantly less, i.e., worse "social functioning" than males $(69.5 \pm 23.8$ and $66.5 \pm 23.0$, respectively, $\mathrm{p}<0.001)$. Moreover, participants who had chronic diseases had significantly less, i.e., worse "bodily pain" than those who did not have chronic diseases (67.7 \pm 25.1 and $77.4 \pm 22.8$, respectively, $\mathrm{p}=0.003)$. However, the "bodily pain" subscale did not differ significantly according to participants' age, nationality, marital status or number of children. "bodily pain" subscale differed significantly according to participants' experience in PHC, being lowest among those with $20+$ years' experience and highest among those with $<5$ years' experience $(67.1 \pm 25.9$ and 80.6 \pm 18.3 , respectively, $\mathrm{p}=0.002$ ). However, the "emotional wellbeing" subscale did not differ significantly according to participants' geographical sector, position or monthly income. The subscale of "role limitations due to physical health" did not differ significantly according to participants' personal characteristics. Participants "role limitations due to physical health" differed significantly according to their monthly income, being best attained by those who had 25,000-29,999 SR, 10,000-14,999 SR, and those who had $\geq 30,000$ SR $(62.5 \pm 42.0,62.6 \pm 39.9$ and $73.4 \pm 39.2$, respectively, $\mathrm{p}=0.013$ ). However, this subscale did not differ significantly according to geographical sector, participants' position, or their experience in PHC. Participants who had chronic diseases had significantly less, i.e., worse " general health" than those who did not have chronic diseases $(56.4 \pm 16.4$ and $64.3 \pm 14.7$, respectively, $\mathrm{p}<0.001)$. Moreover, non-Saudi participants had significantly less, i.e., worse "general health" than Saudi participants $(55.7 \pm 15.6$ and $62.7 \pm 15.5$, respectively, $\mathrm{p}=0.007)$. In addition, the "general health" subscale differed significantly according to their marital status, being lowest (i.e., worse) among divorced participants and highest (i.e., best) among single participants $(55.6 \pm 18.2$ and $63.9 \pm 14.3$, respectively, $\mathrm{p}=0.016$ ). However, participants' "general health" subscale did not differ significantly according to their gender, age, or number of children. The "general health" subscale differed significantly according to participants' position, being lowest (i.e., worst) among dentistry assistants and highest (i.e., best) among dentists (57.5 \pm 12.5 and $65.3 \pm 17.4$, respectively, $\mathrm{p}=0.037)$. However, the "general health" subscale did not differ significantly according to participants' geographical sector, monthly income or experience in PHC.

Table 4: Participants' mean percent scores of "physical functioning", "bodily pain", "role limitations due to physical health", and "general health" according to their personal and work characteristics

\begin{tabular}{|c|c|c|c|c|c|c|c|c|c|}
\hline \multirow{2}{*}{$\begin{array}{c}\text { Personal and } \\
\text { work } \\
\text { characteristics }\end{array}$} & \multirow[t]{2}{*}{ No. } & \multicolumn{2}{|c|}{ Physical functioning } & \multicolumn{2}{|c|}{ Bodily pain } & \multicolumn{2}{|c|}{$\begin{array}{c}\text { Role limitations due } \\
\text { to physical health }\end{array}$} & \multicolumn{2}{|c|}{ General health } \\
\hline & & Mean \pm SD & P-value & Mean \pm SD & P-value & Mean \pm SD & P-value & Mean \pm SD & P-value \\
\hline \multicolumn{10}{|l|}{ Gender } \\
\hline - $\quad$ Male & 213 & $65.1 \pm 29.3$ & & $77.4 \pm 22.8$ & & $61.6 \pm 40.1$ & & $63.2 \pm 15.8$ & \\
\hline - $\quad$ Female & 276 & $63.5 \pm 27.1$ & 0.539 & $69.5 \pm 23.8$ & $<0.001$ & $56.4 \pm 41.0$ & 0.162 & $61.4 \pm 15.4$ & 0.197 \\
\hline \multicolumn{10}{|l|}{ Age } \\
\hline - $\quad<25$ years & 23 & $67.2 \pm 32.9$ & & $84.2 \pm 19.9$ & & $68.5 \pm 40.0$ & & $65.0 \pm 15.0$ & \\
\hline - 25-35 years & 250 & $66.0 \pm 28.0$ & & $740 \pm 22.6$ & & $61.1 \pm 39.6$ & & $63.2 \pm 14.3$ & \\
\hline - $36-45$ years & 148 & $63.6 \pm 28.6$ & & $71.4 \pm 24.7$ & & $57.3 \pm 42.1$ & & $61.5 \pm 17.6$ & \\
\hline - $46-55$ years & 56 & $56.3 \pm 24.2$ & & $67.8 \pm 24.3$ & & $48.7 \pm 40.3$ & & $59.3 \pm 15.2$ & \\
\hline - $\quad>55$ years & 12 & $63.8 \pm 26.8$ & 0.214 & $72.9 \pm 30.1$ & 0.061 & $54.2 \pm 43.7$ & 0.203 & $56.3 \pm 16.7$ & 0.212 \\
\hline \multicolumn{10}{|l|}{ Nationality } \\
\hline - $\quad$ Saudi & 451 & $64.0 \pm 28.2$ & & $73.2 \pm 23.6$ & & $59.5 \pm 40.1$ & & $62.7 \pm 15.5$ & \\
\hline - $\quad$ Non-Saudi & 38 & $65.9 \pm 25.9$ & 0.691 & $70.5 \pm 24.5$ & 0.500 & $49.3 \pm 41.3$ & 0.140 & $55.7 \pm 15.6$ & 0.007 \\
\hline \multicolumn{10}{|l|}{ Marital status } \\
\hline - Single & 88 & $66.1 \pm 31.4$ & & $76.3 \pm 22.9$ & & $61.9 \pm 39.2$ & & $63.9 \pm 14.3$ & \\
\hline - $\quad$ Married & 347 & $64.0 \pm 27.4$ & & $72.5 \pm 23.5$ & & $59.1 \pm 40.5$ & & $62.7 \pm 15.1$ & \\
\hline - $\quad$ Divorced & 44 & $63.6 \pm 26.4$ & & $71.7 \pm 24.1$ & & $51.1 \pm 43.5$ & & $55.6 \pm 18.2$ & \\
\hline - Widow & 10 & $55.0 \pm 26.0$ & 0.677 & $63.3 \pm 32.6$ & 0.299 & $50.0 \pm 45.6$ & 0.465 & $57.5 \pm 23.4$ & 0.016 \\
\hline \multicolumn{10}{|l|}{$\begin{array}{l}\text { Number of } \\
\text { children }\end{array}$} \\
\hline$\cdot 0$ & 148 & $65.8 \pm 29.1$ & & $75.2 \pm 22.5$ & & $62.8 \pm 39.9$ & & $62.7 \pm 14.8$ & \\
\hline $\begin{array}{ll} & 1 \\
\end{array}$ & 72 & $67.4 \pm 27.2$ & & $75.2 \pm 22.0$ & & $57.6 \pm 39.5$ & & $61.4 \pm 16.0$ & \\
\hline $\begin{array}{l}-2 \\
\end{array}$ & 84 & $63.5 \pm 30.3$ & & $72.4 \pm 25.6$ & & $61.3 \pm 40.8$ & & $61.8 \pm 16.5$ & \\
\hline - 3 & 96 & $63.4 \pm 28.4$ & & $73.5 \pm 24.8$ & & $59.6 \pm 40.5$ & & $62.9 \pm 15.9$ & \\
\hline - $4+$ & 89 & $60.3 \pm 24.1$ & 0.524 & $67.3 \pm 23.4$ & 0.129 & $49.2 \pm 42.2$ & 0.142 & $61.5 \pm 15.6$ & 0.940 \\
\hline \multicolumn{10}{|l|}{ Chronic diseases } \\
\hline - $\quad$ Absent & 537 & $66.1 \pm 28.3$ & & $74.9 \pm 22.9$ & & $59.6 \pm 40.1$ & & $64.3 \pm 14.7$ & \\
\hline - $\quad$ Present & 131 & $59.0 \pm 26.5$ & 0.013 & $67.7 \pm 25.1$ & 0.003 & $56.1 \pm 42.3$ & 0.396 & $56.4 \pm 16.4$ & $<0.001$ \\
\hline \multicolumn{10}{|l|}{ Sector } \\
\hline - $\quad$ Northeastern & 118 & $63.9 \pm 27.7$ & & $73.8 \pm 22.5$ & & $58.1 \pm 40.4$ & & $63.1 \pm 13.3$ & \\
\hline - $\quad$ Northwestern & 96 & $68.5 \pm 27.8$ & & $74.2 \pm 22.7$ & & $65.4 \pm 41.3$ & & $64.1 \pm 20.0$ & \\
\hline
\end{tabular}




\begin{tabular}{|c|c|c|c|c|c|c|c|c|c|}
\hline - $\quad$ Southeastern & 81 & $63.2 \pm 25.1$ & & $76.1 \pm 22.3$ & & $62.7 \pm 34.5$ & & $59.9 \pm 16.1$ & \\
\hline - $\quad$ Southwestern & 98 & $66.1 \pm 30.4$ & & $72.7 \pm 25.8$ & & $59.4 \pm 42.6$ & & $61.0 \pm 13.6$ & \\
\hline - $\quad$ Central & 96 & $59.1 \pm 28.1$ & 0.193 & $68.3 \pm 24.6$ & 0.236 & $48.7 \pm 42.0$ & 0.055 & $62.2 \pm 14.5$ & 0.379 \\
\hline \multicolumn{10}{|l|}{ Position } \\
\hline - $\quad$ Physician & 101 & $64.8 \pm 29.8$ & & $73.7 \pm 22.4$ & & $57.4 \pm 43.8$ & & $64.3 \pm 15.8$ & \\
\hline - $\quad$ Dentist & 32 & $62.5 \pm 30.1$ & & $73.5 \pm 23.5$ & & $54.7 \pm 41.4$ & & $65.3 \pm 17.4$ & \\
\hline - $\quad$ Pharmacist & 28 & $71.6 \pm 28.6$ & & $75.0 \pm 23.8$ & & $49.1 \pm 38.8$ & & $63.6 \pm 13.5$ & \\
\hline - $\quad$ Technician & 83 & $67.2 \pm 27.3$ & & $77.4 \pm 22.6$ & & $68.4 \pm 38.5$ & & $63.7 \pm 15.5$ & \\
\hline - $\quad$ Nurse & 143 & $65.0 \pm 26.0$ & & $68.7 \pm 24.4$ & & $55.6 \pm 40.7$ & & $58.6 \pm 16.4$ & \\
\hline - $\begin{array}{l}\text { Dentistry } \\
\text { assistant }\end{array}$ & 14 & $66.4 \pm 24.7$ & & $64.5 \pm 25.4$ & & $48.2 \pm 41.0$ & & $57.5 \pm 12.5$ & \\
\hline $\begin{array}{ll}- & \text { Administrato } \\
\mathrm{r}\end{array}$ & 88 & $57.3 \pm 29.0$ & 0.196 & $74.9 \pm 23.9$ & 0.100 & $62.2 \pm 38.3$ & 0.168 & $63.2 \pm 13.7$ & 0.037 \\
\hline \multicolumn{10}{|l|}{$\begin{array}{l}\text { Monthly income } \\
\text { (SR) }\end{array}$} \\
\hline - $\quad<10,000$ & 95 & $61.1 \pm 32.1$ & & $74.4 \pm 24.2$ & & $60.5 \pm 38.7$ & & $62.9 \pm 14.0$ & \\
\hline $\begin{array}{ll}-10,000- \\
& 14,999 \\
\end{array}$ & 186 & $66.9 \pm 25.4$ & & $74.2 \pm 22.5$ & & $62.6 \pm 39.9$ & & $62.1 \pm 15.4$ & \\
\hline $\begin{array}{ll}-15,000- \\
& 19,999 \\
\end{array}$ & 123 & $67.4 \pm 26.4$ & & $71.3 \pm 24.3$ & & $56.9 \pm 40.7$ & & $61.8 \pm 16.2$ & \\
\hline $\begin{array}{ll}-\quad 20,000- \\
& 24,999 \\
\end{array}$ & 57 & $55.5 \pm 28.5$ & & $69.6 \pm 24.1$ & & $41.7 \pm 43.1$ & & $59.0 \pm 17.1$ & \\
\hline $\begin{array}{ll}- & 25,000- \\
& 29,999 \\
\end{array}$ & 12 & $52.9 \pm 33.9$ & & $76.3 \pm 23.8$ & & $62.5 \pm 42.0$ & & $64.6 \pm 12.7$ & \\
\hline - $\quad \geq 30,000$ & 16 & $65.0 \pm 31.1$ & 0.034 & $72.2 \pm 28.1$ & 0.726 & $73.4 \pm 39.2$ & 0.013 & $70.0 \pm 15.9$ & 0.228 \\
\hline \multicolumn{10}{|l|}{$\begin{array}{l}\text { Experience in } \\
\text { PHC }\end{array}$} \\
\hline - $\quad<5$ years & 95 & $29.1 \pm 29.1$ & & $80.6 \pm 18.3$ & & $65.3 \pm 38.3$ & & $64.5 \pm 14.6$ & \\
\hline - 5-9 years & 171 & $28.3 \pm 28.3$ & & $71.9 \pm 23.5$ & & $61.0 \pm 40.5$ & & $61.1 \pm 16.6$ & \\
\hline - $\quad 10-19$ years & 144 & $27.3 \pm 27.3$ & & $72.4 \pm 24.7$ & & $55.4 \pm 42.6$ & & $61.8 \pm 16.0$ & \\
\hline - $20+$ years & 79 & $26.9 \pm 28.0$ & 0.086 & $67.1 \pm 25.9$ & 0.002 & $51.9 \pm 39.4$ & 0.103 & $62.3 \pm 13.6$ & 0.371 \\
\hline
\end{tabular}

Table 5 shows that participants who had chronic diseases had significantly less "vitality" than those who did not have chronic diseases $(53.7 \pm 20.4$ and $57.6 \pm 17.8$, respectively, $\mathrm{p}=0.041$ ). Moreover, female participants had significantly less "vitality" than males $(53.5 \pm 18.9$ and $60.5 \pm 17.4$, respectively, $\mathrm{p}<0.001)$. However, the "vitality" subscale did not differ significantly according to participants' age, nationality, marital status or number of children. The "vitality" subscale differed significantly according to participants' position, being lowest among dentistry assistants and highest among technicians (44.7 \pm 18.9 and $63.0 \pm 17.5$, respectively, $\mathrm{p}=0.001$ ). However, the "vitality" subscale did not differ significantly according to participants' geographical sector, monthly income or experience in PHC. Participants who had chronic diseases had significantly less "social functioning" than those who did not have chronic diseases $(62.8 \pm 22.2$ and $70.4 \pm 22.1$, respectively, $\mathrm{p}=0.001$ ). Moreover, female participants had significantly less "social functioning" than males $(66.5 \pm 23.0$ and 66.5 \pm 23.0 , respectively, $\mathrm{p}=0.040$ ). However, the "social functioning" component did not differ significantly according to participants' age, nationality, marital status or number of children. Tthe component of "social functioning" did not differ significantly according to participants' work characteristics. Table (20) shows that participants who had chronic diseases had significantly less " role limitations due to emotional problems " than those who did not have chronic diseases $(48.3 \pm 41.0$ and $57.6 \pm 43.0$, respectively, $\mathrm{p}=0.033$ ). However, the "physical functioning" subscale did not differ significantly according to participants' gender, age, nationality, marital status or number of children. Participants " role limitations due to emotional problems" differed significantly according to their geographical sector, being best among participants at Northwestern sector and least among participants at Southeastern sector $(67.7 \pm 43.9$ and 49.4 \pm 37.3 , respectively, $p=0.014)$. However, this component did not differ significantly according to participants' position, monthly income, or their experience in PHC. Participants who had chronic diseases had significantly less "emotional wellbeing" than those who did not have chronic diseases $(60.8 \pm 20.5$ and $64.7 \pm 17.3$, respectively, $\mathrm{p}=0.033$ ). Moreover, female participants had significantly less "emotional wellbeing" than males (62.1 \pm 18.9 and $65.8 \pm 17.3$, respectively, $\mathrm{p}=0.017$ ). However, the "physical functioning" component did not differ significantly according to participants' age, nationality, marital status or number of children. The "emotional wellbeing" subscale differed significantly according to participants' position, being lowest among dentistry assistants and highest among technicians $(58.6 \pm 12.4$ and $68.1 \pm 17.2$, respectively, $\mathrm{p}=0.016$ ). However, the "emotional wellbeing" subscale did not differ significantly according to participants' geographical sector, monthly income or experience in PHC. 
Table 5: Participants' mean percent scores of "vitality", "social functioning", "role limitations due to emotional problems", and "emotional wellbeing" according to their personal and work characteristics

\begin{tabular}{|c|c|c|c|c|c|c|c|c|c|}
\hline \multirow{2}{*}{$\begin{array}{c}\begin{array}{c}\text { Personal and work } \\
\text { characteristics }\end{array} \\
\end{array}$} & \multirow[b]{2}{*}{ No. } & \multicolumn{2}{|c|}{ Vitality } & \multicolumn{2}{|c|}{ Social functioning } & \multicolumn{2}{|c|}{$\begin{array}{l}\text { Role limitations due to } \\
\text { emotional problems }\end{array}$} & \multicolumn{2}{|c|}{ Emotional wellbeing } \\
\hline & & Mean \pm SD & P-value & Mean \pm SD & P-value & Mean \pm SD & P-value & Mean \pm SD & P-value \\
\hline \\
\hline - Male & 213 & $60.5 \pm 17.4$ & & $70.7 \pm 21.2$ & & $55.6 \pm 42.0$ & & $65.8 \pm 17.3$ & \\
\hline - $\quad$ Female & 276 & $53.5 \pm 18.9$ & $<0.001$ & $66.5 \pm 23.0$ & 0.040 & $54.8 \pm 43.1$ & 0.852 & $62.1 \pm 18.9$ & 0.017 \\
\hline \multicolumn{10}{|l|}{ Age } \\
\hline - $\quad<25$ years & 23 & $58.6 \pm 14,9$ & & $73.9 \pm 21.6$ & & $58.0 \pm 47.4$ & & $64.2 \pm 16.8$ & \\
\hline - $25-35$ years & 250 & $57.8 \pm 18.9$ & & $68.7 \pm 22.9$ & & $58.5 \pm 42.8$ & & $64.3 \pm 18.8$ & \\
\hline - $\quad 36-45$ years & 148 & $55.3 \pm 19.0$ & & $66.5 \pm 21.8$ & & $52.5 \pm 42.6$ & & $62.5 \pm 18.0$ & \\
\hline - $46-55$ years & 56 & $54.7 \pm 16.6$ & & $67.4 \pm 19.2$ & & $44.6 \pm 39.3$ & & $62.5 \pm 18.5$ & \\
\hline - $\quad>55$ years & 12 & $51.0 \pm 22.0$ & 0.443 & $78.1 \pm 29.3$ & 0.294 & $61.1 \pm 39.8$ & 0.206 & $69.0 \pm 12.3$ & 0.712 \\
\hline \multicolumn{10}{|l|}{ Nationality } \\
\hline - $\quad$ Saudi & 451 & $56.9 \pm 18.6$ & & $68.8 \pm 22.4$ & & $55.5 \pm 42.6$ & & $64.0 \pm 18.6$ & \\
\hline - $\quad$ Non-Saudi & 38 & $52.0 \pm 17.7$ & 0.114 & $62.8 \pm 20.2$ & 0.112 & $50.9 \pm 42.3$ & 0.520 & $59.6 \pm 14.0$ & 0.148 \\
\hline \multicolumn{10}{|l|}{ Marital status } \\
\hline - $\quad$ Single & 88 & $55.3 \pm 17.8$ & & $69.5 \pm 23.5$ & & $59.8 \pm 42.9$ & & $61.1 \pm 18.6$ & \\
\hline - $\quad$ Married & 347 & $57.2 \pm 18.3$ & & $68.3 \pm 21.8$ & & $54.9 \pm 42.3$ & & $64.9 \pm 17.7$ & \\
\hline - $\quad$ Divorced & 44 & $56.3 \pm 20.4$ & & $67.9 \pm 23.4$ & & $48.5 \pm 44.0$ & & $60.0 \pm 20.4$ & \\
\hline - Widow & 10 & $46.5 \pm 25.0$ & 0.296 & $63.8 \pm 26.0$ & 0.882 & $50.0 \pm 46.1$ & 0.513 & $58.9 \pm 23.8$ & 0.125 \\
\hline \multicolumn{10}{|l|}{ Number of children } \\
\hline - 0 & 148 & $56.1 \pm 17.3$ & & $69.8 \pm 21.9$ & & $58.8 \pm 43.4$ & & $63.5 \pm 18.4$ & \\
\hline $\begin{array}{ll}-1 \\
\end{array}$ & 72 & $59.1 \pm 17.8$ & & $69.4 \pm 20.9$ & & $56.5 \pm 42.5$ & & $62.2 \pm 18.3$ & \\
\hline - 2 & 84 & $59.3 \pm 19.5$ & & $68.8 \pm 22.9$ & & $57.1 \pm 41.6$ & & $65.3 \pm 19.1$ & \\
\hline $\begin{array}{ll} & 3 \\
\end{array}$ & 96 & $54.8 \pm 21.1$ & & $67.4 \pm 23.8$ & & $55.6 \pm 43.2$ & & $63.0 \pm 21.0$ & \\
\hline $\begin{array}{ll}-4+ \\
\end{array}$ & 89 & $54.6 \pm 17.3$ & 0.265 & $65.6 \pm 22.2$ & 0.667 & $45.7 \pm 41.3$ & 0.220 & $64.3 \pm 13.9$ & 0.852 \\
\hline \multicolumn{10}{|l|}{ Chronic diseases } \\
\hline - $\quad$ Absent & 537 & $57.6 \pm 17.8$ & & $70.4 \pm 22.1$ & & $57.6 \pm 43.0$ & & $64.7 \pm 17.3$ & \\
\hline - $\quad$ Present & 131 & $53.7 \pm 20.4$ & 0.041 & $62.8 \pm 22.2$ & 0.001 & $48.3 \pm 41.0$ & 0.033 & $60.8 \pm 20.5$ & 0.033 \\
\hline \multicolumn{10}{|l|}{ Sector } \\
\hline $\begin{array}{ll}- & \text { Northeastern }\end{array}$ & 118 & $58.4 \pm 15.6$ & & $67.8 \pm 20.8$ & & $50.0 \pm 43.7$ & & $64.2 \pm 16.7$ & \\
\hline - $\quad$ Northwestern & 96 & $54.9 \pm 21.5$ & & $69.4 \pm 25.3$ & & $67.7 \pm 43.9$ & & $62.2 \pm 20.5$ & \\
\hline $\begin{array}{ll}\text { - } & \text { Southeastern }\end{array}$ & 81 & $58.2 \pm 18.3$ & & $68.1 \pm 21.1$ & & $49.4 \pm 37.3$ & & $65.2 \pm 17.2$ & \\
\hline $\begin{array}{ll} & \text { Southwestern }\end{array}$ & 98 & $53.7 \pm 18.8$ & & $69.3 \pm 23.6$ & & $57.1 \pm 42.5$ & & $61.1 \pm 19.3$ & \\
\hline - $\quad$ Central & 96 & $57.5 \pm 18.8$ & 0.281 & $67.3 \pm 20.9$ & 0.956 & $51.7 \pm 42.1$ & 0.014 & $65.9 \pm 17.5$ & 1.186 \\
\hline \multicolumn{10}{|l|}{ Position } \\
\hline - $\quad$ Physician & 101 & $54.9 \pm 19.4$ & & $68.9 \pm 22.0$ & & $57.1 \pm 45.5$ & & $60.7 \pm 18.9$ & \\
\hline - $\quad$ Dentist & 32 & $58.1 \pm 16.0$ & & $62.9 \pm 23.6$ & & $57.3 \pm 43.4$ & & $66.2 \pm 20.8$ & \\
\hline - $\quad$ Pharmacist & 28 & $57.9 \pm 18.1$ & & $70.1 \pm 22.9$ & & $47.6 \pm 45.7$ & & $66.4 \pm 18.4$ & \\
\hline - $\quad$ Technician & 83 & $63.0 \pm 17.5$ & & $73.9 \pm 19.8$ & & $59.0 \pm 41.4$ & & $68.1 \pm 17.2$ & \\
\hline - $\quad$ Nurse & 143 & $53.1 \pm 18.9$ & & $64.9 \pm 22.9$ & & $51.3 \pm 41.6$ & & $60.9 \pm 18.9$ & \\
\hline $\begin{array}{ll}- & \begin{array}{l}\text { Dentistry } \\
\text { assistant }\end{array} \\
\end{array}$ & 14 & $44.7 \pm 18.9$ & & $63.4 \pm 28.4$ & & $42.9 \pm 46.1$ & & $58.6 \pm 12.4$ & \\
\hline - $\quad$ Administrator & 88 & $58.9 \pm 17.1$ & 0.001 & $70.2 \pm 21.5$ & 0.062 & $59.1 \pm 40.0$ & 0.551 & $66.5 \pm 16.0$ & 0.016 \\
\hline \multicolumn{10}{|l|}{ Monthly income (SR) } \\
\hline - $\quad<10,000$ & 95 & $59.1 \pm 18.1$ & & $68.8 \pm 23.8$ & & $54.7 \pm 40.9$ & & $65.0 \pm 18.5$ & \\
\hline - $10,000-14,999$ & 186 & $56.9 \pm 19.0$ & & $69.8 \pm 21.1$ & & $59.0 \pm 42.2$ & & $64.3 \pm 18.9$ & \\
\hline - $15,000-19,999$ & 123 & $54.9 \pm 19.4$ & & $65.8 \pm 23.6$ & & $50.7 \pm 43.6$ & & $62.8 \pm 18.9$ & \\
\hline - $20,000-24,999$ & 57 & $53.8 \pm 18.2$ & & $65.4 \pm 21.4$ & & $49.7 \pm 42.8$ & & $61.9 \pm 16.5$ & \\
\hline $\begin{array}{ll}-25,000-29,999 \\
\end{array}$ & 12 & $55.3 \pm 12.9$ & & $71.9 \pm 19.3$ & & $44.4 \pm 49.9$ & & $60.9 \pm 13.7$ & \\
\hline - $\quad>30,000$ & 16 & $60.3 \pm 13.5$ & 0.436 & $76.6 \pm 20.9$ & 0.305 & $75.0 \pm 47.5$ & 0.155 & $63.1 \pm 13.9$ & 0.884 \\
\hline \multicolumn{10}{|l|}{ Experience in PHC } \\
\hline - $\quad<5$ years & 95 & $56.8 \pm 17.6$ & & $70.0 \pm 21.8$ & & $57.9 \pm 43.3$ & & $64.4 \pm 18.1$ & \\
\hline - 5-9 years & 171 & $58.3 \pm 19.6$ & & $66.2 \pm 23.3$ & & $59.6 \pm 42.2$ & & $63.2 \pm 19.0$ & \\
\hline - $10-19$ years & 144 & $55.5 \pm 18.5$ & & $70.1 \pm 22.3$ & & $52.1 \pm 43.3$ & & $64.2 \pm 17.3$ & \\
\hline - $20+$ years & 79 & $54.4 \pm 17.6$ & 0.402 & $67.9 \pm 20.7$ & 0.402 & $47.7 \pm 40.5$ & 0.140 & $62.9 \pm 18.8$ & 0.917 \\
\hline
\end{tabular}




\section{Discussion}

Quality of life has been recognized as an important measure in numerous healthy populations. For the provision of satisfactory public health care, it is necessary to have health care workers with a high quality of life (17). Decline in health care workers' quality of life may lead to reduced professional performance, quality, and safety of provided health care services. ${ }^{18}$

Therefore, the present study aimed to assess the quality of life among primary health care workers and to determine predictors of their quality of life.

Results of the current study showed that $\mathrm{MOH}$ primary health care physicians' quality of life in Jeddah City is generally suboptimal. Out of $100 \%$, participants' quality of life physical component summary mean percent score was $64.5 \pm 19.4 \%$, that of mental component summary was $60.9 \pm 17.5 \%$, while their mean percent score of health related quality of life was $62.7 \pm 17.5 \%$. Their best attained health-status scores were for "bodily pain" and "social functioning" subscles $(72.9 \pm 23.7 \%$ and $68.4 \pm 22.3 \%$, respectively), while their least attained health-status scores were for "role limitations due to emotional problems" and "energy/fatigue" ( $58.7 \pm 4.7 \%$ and $56.6 \pm 18.6 \%$, respectively).

These findings are in accordance with those reported by several other studies. Khalooei and Homaei ${ }^{18}$ reported that the mean HRQOL score of family medicine team members was $71.6 \%$. Gholami et al. ${ }^{19}$ found that the nursing staff quality of life mean score was $64.7 \%$. Moreover, Sveinsdóttir and Gunnarsdóttir ${ }^{20}$ demonstrated that healthcare workers' quality of life was not satisfactory. Shanafelt et al., ${ }^{21}$ in USA, reported physicians' quality of life is lower than that among other US workers.

However, Kheiraoui et al., ${ }^{22}$ in Italy, reported that healthcare workers had relatively higher quality of life scores, with the highest attained mean score of $92.8 \pm 11.7$ for "physical health" and "role physical" (78.2 \pm 35.9$)$, while the least scores were for "vitality" $(61.9 \pm 20.7)$ and "general health" $(65.2 \pm 22.3)$.

The identified unfavorable HRQoL among health care workers at $\mathrm{MOH}$ primary health care centers in Jeddah necessitates further studies to explore the possible causative factors leading to their suboptimal quality of life and to put plans for its improvement. Kheiraoui et al. ${ }^{22}$ reported that burnout and increased burden among healthcare workers is responsible for their lowered quality of life. They suggested that minimizing the burden on healthcare personnel is expected to improve their quality of life and medical outcomes of their patients.

Results of the current study showed that some personal characteristics were associated with lower HRQoL scores among primary health care workers. Female gender was significantly associated with lower HRQoL regarding both physical component summary and its bodily pain subscale, as well as the mental component summary and its subscales of vitality, social functioning and emotional wellbeing.

Similarly, Kheiraoui et al. ${ }^{22}$ reported that female healthcare workers had consistently lower scores for all eight subscales of SF-36, with statistically significant differences regarding bodily pain $(\mathrm{p}=0.005)$, vitality $(\mathrm{p}=0.008)$ and mental health $(\mathrm{p}=0.015)$. They explained this common finding by being related to the responsibilities of women outside of work (child care, domestic and family commitments).

Liang et al. ${ }^{23}$ added that it has been observed that health of female physicians is usually poorer than that of males. For example, female doctors suffer from more minor physical ailments and higher suicidal rate than male doctors. ${ }^{24}$ Therefore, health of female health care providers should be considered and a support-network system should be developed. ${ }^{25,26}$

It was not surprising to realize in the present study that primary health care workers with chronic diseases had consistently and significantly lower scores in all components and subscales of HRQoL than those who did not have chronic disease.

Lam and Lauder ${ }^{27}$ noted that advances in medicine have prolonged the life of many people with chronic diseases, which may not kill but rather threaten the quality of life of sufferers.

The present study also identified few other personal characteristics as determinants of some components and subscales of HRQoL among primary health care workers. Older participants had significantly lower scores for "physical component summary" than younger ones. NonSaudi participants had significantly lower scores for "general health" subscale than Saudi ones. Divorced/single participants had significantly lower scores for "general health" subscale than others.

Almalki et al., ${ }^{28}$ in Jazan Region, Saudi Arabia, reported that significant differences were associated with nurses' HRQoL were their gender, age, marital status, dependent children, dependent adults, nationality, and payment per month.

Results of the current study showed that some workrelated characteristics were also associated with lower HRQoL scores among primary health care workers. HRQoL components and subscales that were significantly associated with the geographic location of sectors, i.e., "physical component summary", "role limitations due to emotional problems" subscale, with "Central" being associated with significantly lower scores. Dentistry assistants were consistently and significantly associated with low scores for "general health"; "vitality"; "emotional wellbeing" subscales; "mental component summary" component and the "health-related quality of life". Participants with relatively lower monthly income had significantly lower HRQoL scores regarding "physical component summary"; "physical functioning" and "role limitations due to physical health" subscales. Participants' longer experience in PHC was significantly associated with lower HRQoL scores regarding both "physical component summary" component and "bodily pain" subscale.

Regarding the significant impact of age on health care providers' quality of life, Baldwin et al. ${ }^{29}$ noted that older people feel more pain than younger ones. However, the research objects of the study are young clinical doctors. 
Therefore, young doctors unlikely suffer from senile and chronic diseases.

The study of Liang et al., ${ }^{23}$ in China, reported that risk factors for poor quality of life among health care providers' include being a female, lower job title or position, and lower salary. Sonnentag and Zijlstra ${ }^{30}$ stressed that, with high wages, individuals' material life can be guaranteed, and QOL can be improved. Thus, the government should also strengthen the financial support for health care providers. ${ }^{31}$

It is to be noted that, personal and work-related characteristics that are significantly related to lower quality of life, (e.g., female gender, lower salary, working at Central PHC center, being a dentistry assistant, having a longer PHC experience, etc.) necessitate further study to identify the cause(s) for lowered HRQoL among primary health care providers and to suggest the proper strategy that can be used by primary health care managers and policy makers at the Saudi MOH for developing and appropriately implementing successful plans to improve their HRQoL.

\section{Conclusions}

Based on findings of the present study, it can be concluded that health related quality of life (HRQoL) of primary health care providers in Jeddah City is suboptimal. The mental component of HRQoL is lower than the physical component. Some subscales of HRQoL are specially low, e.g., Role limitations due to emotional problems or due to physical health and vitality. The main determinants of low HRQoL that are associated with primary care providers' personal characteristics include being a female, older age, and having a chronic disease, while those associated with primary care providers' work characteristics include serving at "Central" PHC, being a dentistry assistant.

The researchers recommended: explore the cause(s) for lowered HRQoL among primary health care providers, especially those with significant risk factors (i.e., females, those working in Central PHCC, those with chronic diseases, etc.). Conduct further studies to assess HRQoL of primary care providers in other areas of the Kingdom of Saudi Arabia and to explore more risk factors associated with low HRQoL. And propose the proper strategy for developing and implementing the necessary plans to improve HRQoL among PHC providers.

\section{Conflict of Interest: None.}

\section{Abbreviations}

ANOVA: One way-analysis of variance

CI: Confidence interval.

GP: GENERAL practitioner.

HRQoL: Health related quality of life

HCW: Health Care Workers

IM-ITE: Internal Medicine In-Training Examination

MOH: Ministry Of Health

OR: Odds ratio

PHCC: Primary Health Care Centres.

QOL: Quality of life.

SPSS: Statistical Package for Social Sciences

\section{References}

1. Muldoon MF, Barger SD, Flory JD, Manuck SB. What are quality of life measurements measuring? $\mathrm{Br} \mathrm{Med} J$. 1998;316(7130):542-545.

2. Noor SM, Abdullah MA. Quality Work Life among Factory Workers in Malaysia. Procedia - Soc Behav Sci. [Internet]. 2012;35(December 2011):739-745. Available from: http://dx.doi.org/10.1016/j.sbspro.2012.02.144

3. Burckhardt CS, Anderson KL. The Quality of Life Scale (QOLS): reliability, validity, and utilization. Health Qual Life Outcomes [Internet]. 2003 Oct 23 [cited 2016 Dec 23];1:60. Available from:

http://www.ncbi.nlm.nih.gov/pubmed/14613562

4. Borglin G, Edberg AK, Rahm Hallberg I. The experience of quality of life among older people. J Aging Stud. 2005;19(2):201-220.

5. Diener E, Suh E. Measuring quality of life: economic, social, and subjective indicators. Soc Indic Res [Internet]. 1997 [cited 2016 Dec 20];40(1/2):189-216. Available from: http://link.springer.com/10.1023/A:1006859511756

6. Felce D, Perry J. Quality of life: its definition and measurement. Res Dev Disabil. 1995;16(1):51-74.

7. 36-Item Short Form Survey (SF-36) Scoring Instructions | RAND [Internet]. Available from:

http://www.rand.org/health/surveys_tools/mos/36-item-shortform/scoring.html

8. Carr AJ, Thompson PW, Kirwan JR. Quality of life measures. Br J Rheumatol. 1996;35:275-281.

9. Whoqol Group. The World Health Organization Quality of Life assessment (WHOQOL): position paper from the World Health Organization. Soc Sci Med. 1995;41(10):1403-1409.

10. Greenhaus JH, Collins KM, Shaw JD. The relation between work-family balance and quality of life. $J$ Vocat Behav. 2003;63(3):510-531.

11. Gill TM, Feinstein a R. A critical appraisal of the quality of quality-of-life measurements. JAMA. 1994;272(8):619-626.

12. Rezende GL, Mello MSMS, Granjeiro RC, Nakanishi M, Oliveira CAPC de. The quality of life among

Otorhinolaryngology residents in Distrito Federal (Brazil). Brazilian J Otorhinolaryngol [Internet]. 2011 Aug [cited 2016 Dec 29];77(4):466-472. Available from: http://www.scielo.br/scielo.php?script=sci_arttext\&pid=S1808 $-86942011000400010 \& \operatorname{lng}=$ en\&nrm=iso\&tlng=en

13. Sangi-Haghpeykar H, Ambani DS, Carson SA. Stress, workload, sexual well-being and quality of life among physician residents in training. Int J Clin Pract. 2009;63(3):462-467.

14. Raosoft Sample Size Calculator. Website: http://www.raosoft.com/samplesize.html. Accessed: January 2018.

15. Montazeri A, Goshtasebi A, Vahdaninia M, Gandek B. The Short Form Health Survey (SF-36): translation and validation study of the Iranian version. Qual Life Res. 2005;14(3):875882.

16. Motamed N, Ayatollahi AR, Zare N, Sadeghi-Hassanabadi A. Validity and reliability of the Persian translation of the SF-36 version 2 questionnaire. East Mediterr Health J. 2005;11(3):349-357.

17. Teles MAB, Barbosa MR, Vargas AMD, Gomes VE, Ferreira EF, Martins AM, Ferreira RC. Psychosocial work conditions and quality of life among primary health care employees: a cross sectional study. Health and Quality of Life Outcomes 2014;12:72.

18. Khalooei A, Homaei A. Health-related quality of life and related factors among members of family medicine teams at Kerman University of Medical Sciences, Iran. SDH. 2017;3(2):70-77. 
19. Gholami A, Farsi M, Hashemi Z, Lotfabadi P. Quality of life in nurses working in Neyshabur hospitals. Thrita. 2013;2(1):94-100

20. Sveinsdóttir H, Gunnarsdóttir HK. Predictors of self-assessed physical and mental health of Icelandic nurses: results from a national survey. Int J Nurs Stud. 2008;45(10):1479-1789.

21. Shanafelt TD, Boone S, Tan L, Dyrbye LN, Sotile W, Satele D, West CP, Sloan J, Oreskovich MR. Burnout and Satisfaction With Work-Life Balance Among US Physicians Relative to the General US Population. Arch Intern Med. 2012;172(18):1377-1385.

22. Kheiraoui F, Gualano MR, Mannocci A, Boccia A, La Torre G. Quality of life among healthcare workers: A multicenter cross-sectional study in Italy. Public Health. 2012;126:624629.

23. Liang Y, Wang H, Tao X. Quality of life of young clinical doctors in public hospitals in China's developed cities as measured by the Nottingham Health Profile (NHP). Int J Equity Health. 2015;14:85.

24. Schernhammer ES, Colditz GA. Suicide rates among physicians: a quantitative and gender assessment (metaanalysis). Am J Psychiatry. 2004;161(12):2295-2302.

25. Rout U, Rout R, Rout JK. Stress, mental health and satisfaction among women doctors in England. Eur Psychiatry. 2008;23:S88-S89.

26. Zhou Y, Zhou L, Fu C, Wang Y, Liu Q, Wu H, Zhang R, Zheng L. Socio-economic factors related with the subjective well-being of the rural elderly people living independently in China. Int J Equity Health. 2015;14(1):5.
27. Lam CLK, Lauder IJ. The impact of chronic diseases on the health-related quality of life (HRQOL) of Chinese patients in primary care. Family Practice. 2000;17(2):159-166.

28. Almalki MJ, FitzGerald G, Clark M. Quality of work life among primary health care nurses in the Jazan region, Saudi Arabia: a cross-sectional study. Human Resources for Health. 2012;10:30.

29. Baldwin PJ, Dodd M, Wrate RW. Young doctors' health-I. How do working conditions affect attitudes, health and performance?. Soc Sci Med. 1997;45(1):35-40.

30. Sonnentag S, Zijlstra FR. Job characteristics and off-job activities as predictors of need for recovery, well-being, and fatigue. J Appl Psychol. 2006;91(2):330.

31. Liu Y. China's public health-care system: facing the challenges. Bull World Health Organ. 2004;82(7):532-538.

How to cite this article: Tashkandi D. K, Akber N. A. M. Quality of life among health care workers in primary health care centres in ministry of health Jeddah 2017. J Community Health Manag. 2018;5(4):177-187. 